\title{
Pharmacist-Patient Relationship: Commitment to Care
}

\author{
Abdul Kader Mohiuddin* \\ Faculty of Pharmacy, Dhaka \\ *Corresponding author: Abdul Kader Mohiuddin, Faculty of Pharmacy, WUB, Plot: 14, Lake Drive Road Sector: \\ Sector - 7, Uttara, Dhaka - 1230, Bangladesh
}

\begin{tabular}{|c|c|}
\hline ARTICLE INFO & ABSTRACT \\
\hline Received: 幽 August 28, 2019 & Citation: Abdul Kader Mohiuddin. Pharmacist-Patient Relationship: Commitment to \\
\hline Published: September 03, 2019 & Care. Biomed J Sci \& Tech Res 21(1)-2019. BJSTR. MS.ID.003549. \\
\hline
\end{tabular}

\section{Editorial}

Pharmacy practice has changed substantially in recent years. The professionals have the opportunity to contribute directly to patient care in order to reduce morbimortality related to medication use, promoting health and preventing diseases. Healthcare organizations worldwide are under substantial pressure from increasing patient demand. Unfortunately, a cure is not always possible particularly in this era of chronic diseases, and the role of physicians has become limited to controlling and palliating symptoms. The increasing population of patients with long-term conditions are associated with high levels of morbidity, healthcare costs and GP workloads. This has raised questions about the role of many community pharmacists who traditionally have not had access to important data needed for the first step of the patient care process that defines a valid patient-practitioner relationship. However, community pharmacies are accessible and convenient primary care venues with long opening hours and non-appointment-based services. Community pharmacists are increasingly clinically trained healthcare professionals whose skills and knowledge could be further utilized.

Medical professionals have traditionally abided by a set of ethical tenets including autonomy, justice, beneficence and non-maleficence. Much emphasis is often placed in medicine on non-maleficence, or "do no harm," but the other ethical tenets deserve further consideration when discussing the right-to-refuse legislation. Pharmacists have an obligation to do what is in the best interest of their patients (beneficence), to treat patients justly or fairly (justice), and to respect a patient's decision to know and do what is best for them (autonomy). To put a pharmacist's autonomy above the ethical commitments owed to the patient is fraught with challenges, regardless of legal protections for pharmacists provided through legislation such as the conscience clauses. To transition to patient-centered care, pharmacy services should organize around the understanding of patients' needs, preferences, and expectations for the clinical judgment and decision-making processes.

This is mainly important in cancer care, since the demands of patients are often complex and painful and not necessarily related to the biological aspects of the illness. To preserve patient privacy and confidentiality, consultation rooms became a prerequisite for community pharmacies offering advanced services under the new contract. There are also other medication and public health services which can be commissioned according to local need. These "Locally commissioned services" include minor ailments management, lifestyle advice, blood pressure checks, cholesterol tests and smoking cessation services. These extended services currently provide opportunities for community pharmacists to offer support for patients with long-term care that extends beyond medicines supply. This lack of GP support/awareness also impacts patients' awareness, demand and use of community pharmacy services as many patients seek GPs endorsement for use of healthcare services. A lack of community pharmacy integration within this patient pathway prevents benefits to patients or the healthcare system through the optimal use of extended pharmacy services. It is important to identify how community pharmacies could be better used and integrated within the patient's primary care pathway, as effective collaboration between GPs and community pharmacists will be an important factor to optimize patient care.

The number of patients with multiple diseases requiring multiple medication therapies is increasing steadily. When dealing with these patients, clinical pharmacists must combine several information sources to improve the accuracy of assessment of 
medication adherence. Medication errors may occur in any part of the medication process and are affected by several factors, including the individual patient, the hospital physical environment, and medication-related factors. To reduce the risk of polypharmacy, physicians and clinical pharmacists must collect information on patients' visits to other medical institutions and the prescriptions received there. As patients often lack the information and expertise needed to determine how to properly use various medications, they must completely place their trust in the expert knowledge of clinical pharmacists. Patients with multiple diseases who are at potential risk of drug-drug interactions often place their entire trust in clinical pharmacists. When a patient simultaneously suffers from multiple diseases, the probability of adverse drug reactions from improper or unnecessary medication is high and medical resources may be wasted.

Trust is the degree of confidence that patients possess in their clinical pharmacist's reliability and integrity. Trust is viewed as a comprehensive construct which reflects patient overall trust in clinical pharmacists. To address drug-drug interactions risk, patients rely on the judgment of experts (i.e., clinical pharmacists) in professional systems. Clinical pharmacists can help patients manage their medicines and reduce their anxiety about taking multiple medicines. Pharmacists have unique training and expertise in the appropriate use of medications and provide a wide array of patient care services in many different practice settings. These services reduce adverse drug events, improve patient safety, and optimize medication use and health outcomes. Pharmacists contribute to improving patients' health by providing patient care

\section{ISSN: 2574-1241}

DOI: 10.26717/BJSTR.2019.21.003549

Abdul Kader Mohiuddin. Biomed J Sci \& Tech Res

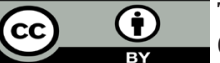

This work is licensed under Creative Commons Attribution 4.0 License

Submission Link: https://biomedres.us/submit-manuscript.php services as authorized under their scope of practice and facilitated by collaborative practice agreements. However, there is variability in how this process is taught and practiced. To promote consistency across the profession, national pharmacy associations used a consensus-based approach to articulate the patient care process for pharmacists to use as a framework for delivering patient care in any practice setting.

If pharmacists and patients agree on relationship roles, the functionality and outcomes of this relationship will be optimized. Future research is needed to monitor trends in pharmacists' and patients' views of their relationship roles and to develop strategies as needed to ensure that pharmacists and patients are following the same relationship script. Pharmacists' professional roles have maturated to include provision of information, education, and pharmaceutical care services. These changes have resulted in a focus on collaborative pharmacist-patient professional relationships, in which pharmacists and patients both have roles and responsibilities. The goal of high quality, cost-effective and accessible health care for patients is achieved through team-based patient-centered care. Pharmacists are essential members of the health care team. The profession of pharmacy is continuing its evolution from a principal focus on medication product distribution to expanded clinically oriented patient care services. As a result of this professional evolution, the importance of, and need for, a consistent process of care in the delivery of patient care services has been increasingly recognized by the profession at large.

\section{Conflict of Interest}

The author declares that he has no competing interests.

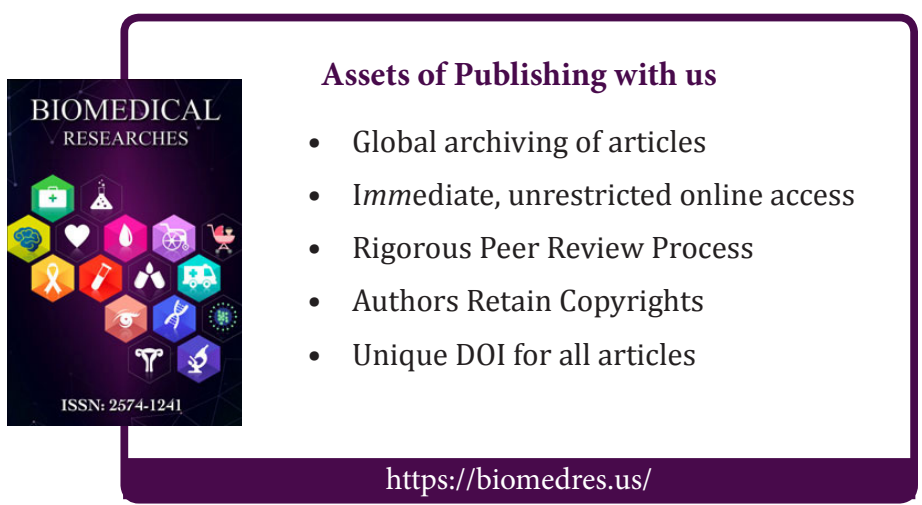

